\title{
Prácticas Clínicas en el Cuidado de Gestantes y Recién Nacidos en Algunos Hospitales de Bogotá, Colombia
}

\author{
Ricardo Rojas-Higuera ${ }^{1}$, Juan G. Londoño-Cardona² y Fernando Arango-Gómez ${ }^{3}$ \\ ${ }^{1}$ Médico Cirujano. Especialización en Gerencia Social. Secretaría Distrital de Salud de Bogotá. E-mail: \\ rrojas@saludcapital.gov.co \\ ${ }^{2}$ Médico Cirujano. Especialización en Ginecología y Obstetricia. Departamento de Obstetricia y Ginecología, \\ Hospital Universitario San Vicente de Paúl de Medellín.E-mail: obstygine@medicina.udea.edu.co \\ ${ }^{3}$ Médico Cirujano. Especialización en Neonatología. Especialización en Pediatría. Facultad de Ciencias \\ para la Salud, Universidad de Caldas. E- mail: farango@telesat.com.co \\ Recibido 29 Septiembre 2005/Enviado para Modificación 25 Enero 2006/Aceptado 21 Septiembre 2006
}

\section{RESUMEN}

Objetivo Evaluar en algunos hospitales de Bogotá, entre Agosto de 2004 y Marzo de 2005, la frecuencia de uso de 17 prácticas en el manejo de las principales causas de morbilidad y mortalidad materna y perinatal.

Métodos Se realizó un estudio de corte transversal. La información corresponde a 3108 mujeres que tuvieron parto con edad gestacional mayor a 24 semanas. Resultados En cada variable evaluada se obtuvieron los siguientes porcentajes: porte del carné perinatal $(79,4 \%)$, suplementación con hierro y ácido fólico $(87,3 \%)$, tamización para sífilis $(69,5 \%)$, tamización para bacteriuria $(69,7 \%)$, acompañamiento continuo durante el trabajo de parto (42,0\%), abstenerse de usar enemas (99,4\%), abstenerse de rasurar el periné (93\%), episiotomía selectiva en primíparas (56,5\%), posición cuclillas o sentada en el parto $(7,9$ $\%)$, manejo activo del trabajo de parto en gestación mayor a 41 semanas $(14,7$ $\%)$, manejo activo del alumbramiento (73,5 \%), administración de antibióticos profilácticos en cesárea $(61,2 \%)$, tratamiento con sulfato de magnesio de la pre-eclampsia severa $(76,5 \%)$ y la eclampsia (20\%), administración de corticoides para inducir maduración fetal en madres con alto riesgo de parto pretérmino (51,6\%); lactancia materna exclusiva y sin restricciones (99,1\%), y alojamiento conjunto madre-recién nacido $(91,2 \%)$.

Conclusiones Se requiere el desarrollo de metodologías efectivas que garanticen la adherencia a las normas y guías de atención materna y perinatal, y de decisiones administrativas y gerenciales que faciliten su cumplimiento.

Palabras Clave: Servicios de salud para maternas, calidad de la atención de salud, acceso, evaluación (fuente: DeCS, BIREME). 


\section{ABSTRACT Clinical practice in looking after breastfeeding women and the new-born in some hospitals in Bogotá, Colombia}

Objective Evaluating the frequency of 17 practices being used in managing the main causes of maternal and perinatal morbidity and mortality in some hospitals in Bogotá between August 2004 and March 2005.

Methods A cross-sectional study was carried out. The data related to 3,108 women who gave birth at gestational age greater than 24 weeks.

Results The following percentages were obtained for each variable evaluated: having a perinatal record card $(79,4 \%)$, taking iron and folic acid supplement $(87,3 \%)$, syphilis screening $(69,5 \%)$, bacteriuria screening $(69,7$ $\%)$, constant accompaniment during giving birth (42,0\%), not taking enemas $(99,4 \%)$, not shaving the perinea $(93,0 \%)$, selective episiotomy in first-time mothers (56,5\%), adopting a squatting or seated position when giving birth $(7,9 \%)$, active management of birth in gestation greater than 41 weeks $(14,7 \%)$, active management of birth $(73,5 \%)$, administering prophylactic antibiotics during a caesarean birth $(61,2 \%)$, treating severe pre-eclampsia $(76,5 \%)$ and eclampsia $(20,0 \%)$ with magnesium sulphate, administering corticoids for inducing foetal maturation in mothers having a high risk of predetermined birth (51,6\%), exclusive maternal lactation, having no restrictions (99,1\%) and joint mother-newborn accommodation (91,2 \%).

Conclusions Effective methodologies are required for guaranteeing adherence to maternal and perinatal attention regulations and guidelines and those administrative and management decisions facilitating compliance with them.

Key Words: Maternal health service, health attention quality, access, evaluation (source: $M e S H, N L M$ ).

$\mathrm{E}$ n la actualidad los profesionales de la salud tenemos posibilidades de acceder a muy buena información acerca de la efectividad de las formas de cuidado que son usadas rutinariamente en la atención de gestantes y recién nacidos. Utilizar esta información como base para las recomendaciones a servicios de salud y la comunidad resultaría en un aumento en el uso de las prácticas beneficiosas y en la erradicación de aquéllas demostradas como perjudiciales. Esto implicaría una mejoría en la calidad de atención a madres y niños a través de una reducción de la morbilidad y mortalidad materna y perinatal, de un aumento en la satisfacción de las usuarias, y de una reducción en los costos de la atención.

El objetivo principal de este estudio fue evaluar la frecuencia de prácticas en el manejo de las principales causas de morbimortalidad materna y perinatal, 
comprobadas científicamente como beneficiosas, inefectivas o perjudiciales, en el cuidado del embarazo, trabajo de parto, parto, puerperio y recién nacido y que pudieran medirse a través del sistema informático perinatal (SIP) diseñado por el Centro Latinoamericano de Perinatología y Desarrollo Humano (CLAP). El estudio se realizó durante ocho meses, en algunos hospitales de la red adscrita a la Secretaría Distrital de Salud de Bogotá, Colombia.

\section{MÉTODOS}

El presente, es un estudio de corte transversal. Se propuso evaluar la frecuencia de uso de prácticas comprobadas científicamente como beneficiosas, inefectivas o perjudiciales, en el cuidado del embarazo, trabajo de parto y parto, puerperio y recién nacido, en algunos hospitales de la ciudad de Bogotá.

Selección de variables

Las fuentes de selección fueron las tablas de prácticas efectivas, inefectivas o perjudiciales publicadas en el libro Guide to Effective Care in Pregnancy and Childbirth (1), y las tablas similares publicadas en la Biblioteca de Salud Reproductiva de la OMS (2). El proceso de selección fue realizado en consenso por los coordinadores de la investigación de los Centros colombianos Asociados al CLAP. Se evaluaron las prácticas que pudieran medirse a través del Sistema Informático Perinatal (SIP), diseñado por el Centro Latinoamericano de Perinatología y Desarrollo Humano (CLAP) (3). Para algunos indicadores se utilizó el programa EPIINFO y SPSSS.

El estudio se realizó en tres hospitales de la red adscrita a la Secretaría Distrital de Salud de Bogotá, los cuales tienen las siguientes características:

Hospital Simón Bolívar, institución de tercer nivel de atención, que desarrolla actividades docentes-asistenciales, con un promedio de 2648 partos al año, el Hospital San Blas institución de segundo nivel de atención, que desarrolla actividades docentes-asistenciales, con un promedio de 1958 partos al año y el Hospital Rafael Uribe Uribe institución de primer nivel de atención, con un promedio de 914 partos al año.

Se incluyeron las mujeres que tuvieron su parto en los hospitales seleccionados. Se definió como parto aquellas terminaciones del embarazo a edad gestacional (EG) mayor o igual a 24 semanas o con recién nacido con peso mayor o igual a $500 \mathrm{~g}$ si la EG no era confiable.

El personal de salud, médicos y enfermeras, que atienden gestantes en los 
hospitales participantes, fueron entrenados desde hace varios años, en el diligenciamiento de la Historia Clínica Materno-Perinatal, adaptada para la captura de la información de la investigación. Con el fin de garantizar el diligenciamiento completo de la historia, incluidas las variables del estudio, se capacitó al personal de las instituciones durante el primer trimestre de 2004.

Del primero de Agosto de 2004 al 27 de Marzo de 2005 se recolectó la información en la Historia Clínica Materna-Perinatal. Esta información fue analizada cada mes por los investigadores principales, con los coordinadores de la atención en salud de cada hospital, y cada dos meses se consolidó la información, se realizaron los controles de llenado y calidad de los datos.

Las prácticas seleccionadas fueron: cuidados durante la gestación: porte del carné perinatal, suplementación con hierro y ácido fólico, tamización para sífilis y bacteriuria asintomática; durante el trabajo de parto: acompañamiento continuo durante el trabajo de parto, abstenerse del uso de enemas y rasurado perineal, episiotomía selectiva en primíparas, posición en el parto, manejo activo del trabajo de parto en gestación mayor a 41 semanas, y manejo activo del alumbramiento; en el tratamiento de complicaciones de la gestación y el parto: administración de antibióticos profilácticos en cesárea, tratamiento de la preeclampsia severa y eclampsia con sulfato de magnesio, y administración de corticoides para inducir maduración pulmonar fetal en madres con alto riesgo de parto pretérmino; en cuidados del recién nacido: lactancia materna exclusiva y sin restricciones, alojamiento conjunto madre-recién nacido.

Aspectos éticos

No se solicitó consentimiento de las gestantes ya que el estudio es de diseño observacional por lo que únicamente se recolectó información sobre eventos del embarazo, del parto y de atención al recién nacido, sin realizar ninguna intervención. La identificación de mujeres y niños quedó resguardada por los procesos definidos por cada institución de salud.

\section{RESULTADOS}

Se presentan los porcentajes de uso de cada una de las prácticas.

Características generales de la población

La información que se presenta corresponde a 3108 mujeres que tuvieron parto a una edad gestacional mayor de 24 semanas de gestación. Dentro de las 
características socio-demográficas de la población participante del estudio se trata de mujeres afiliadas al régimen subsidiado del Sistema de Seguridad Social de Salud en Colombia (42,7 \%) (vinculación que se hace a través del pago de una cotización subsidiada, total o parcialmente, con recursos fiscales o de solidaridad), así como a lo que este sistema denomina pobres sin capacidad de pago o participantes vinculados $(27,8 \%)$ (personas que por motivos de incapacidad de pago y mientras logran ser beneficiarios del régimen subsidiado tendrán derecho a los servicios de atención de salud que prestan las instituciones públicas y aquellas privadas que tengan contrato con el Estado). Un menor porcentaje de mujeres se encontraba afiliada al régimen contributivo $(0,7 \%)$ (vinculación que se hace a través del pago de una cotización, individual y familiar, o un aporte previo financiado directamente por el afiliado o en concurrencia entre éste y su empleador). En general las gestantes atendidas tienen algún nivel educativo principalmente educación secundaria.

Según características de la gestación actual es importante resaltar un promedio de 5 controles prenatales, el inicio del trabajo de parto fue principalmente espontáneo (77,6 \%), seguido por la cesárea electiva (8,6 \%) y la forma inducida (6,6 \%). Las terminación del parto fue espontáneo (77,0 \%), por cesárea (17,1 \%) o con forceps (2,3 \%). La atención del parto y del recién nacido la realizó personal médico en el 99,5\% de los casos.

\section{Prácticas clínicas en el cuidado de gestantes y recién nacidos}

La falta de información para el total de prácticas evaluadas osciló entre el $0,7 \%$ y el 37,9 \%. Esta falta de información por grupos de variables, para cuidados durante la gestación osciló entre el 0,5 \% y el 30,2 \%; de cuidados durante el trabajo de parto varió entre el 0,8 \% y 3,5 \%; del tratamiento de complicaciones de la gestación y el parto estuvo entre el 3,6 \% y el 14,2 \%; y para cuidados del recién nacido entre el 25,8 \% y el 27,3 \%.

El porcentaje de uso de las prácticas de cuidados durante la gestación, el parto, en el tratamiento de complicaciones de la gestación, y en el cuidado del recién nacido, se presentan en las Tablas 1,2,3 y 4. 
228 REVISTA DE SALUD PÚBLICA · Volumen 8 (3), Noviembre 2006

Tabla 1. Prácticas en el cuidado durante la gestación

\begin{tabular}{cccc}
\hline & Prácticas & Frecuencia & $\%$ \\
\hline Porte del carné materno- & No & 559 & 20,6 \\
perinatal & Si & 2149 & 79,4 \\
\hline \multirow{2}{*}{$\begin{array}{c}\text { Suplementación con } \\
\text { hierro y ácido fólico a las } \\
\text { embarazadas }\end{array}$} & Ninguno & 163 & 8,6 \\
& Solo Hierro & 65 & 3,4 \\
Tamización para sífilis & Hierro y Folatos & 14 & 0,7 \\
antes de la semana 20 de & Negativa & 1537 & 87,3 \\
gestación & Positiva & 42 & 67,6 \\
& No se hizo & 694 & 1,9 \\
Tamización para sífilis & Negativa & 1210 & 63,5 \\
después de la semana 20 & Positiva & 45 & 2,4 \\
de gestación & No se hizo & 645 & 34,0 \\
\hline Tamización de bacteriuría & No se hizo & 696 & 30,3 \\
asintomática durante el & Negativa & 1224 & 53,3 \\
embarazo & Positiva & 376 & 16,4 \\
\hline
\end{tabular}

Tabla 2. Prácticas en el cuidado durante el parto y posparto

\begin{tabular}{|c|c|c|c|}
\hline Prácticas & & Frecuencia & $\%$ \\
\hline \multirow{4}{*}{$\begin{array}{l}\text { Acompañamiento continuo } \\
\text { durante el trabajo de parto }\end{array}$} & Pareja & 271 & 11,4 \\
\hline & Familiar & 257 & 10,8 \\
\hline & Otros & 469 & 19,8 \\
\hline & Ninguno & 1375 & 58,0 \\
\hline \multirow{2}{*}{$\begin{array}{l}\text { Enema evacuante durante el } \\
\text { trabajo de parto }\end{array}$} & No & 2460 & 99,4 \\
\hline & Si & 15 & 0,6 \\
\hline \multirow{2}{*}{$\begin{array}{l}\text { Rasurado perineal de rutina } \\
\text { para la preparación del parto }\end{array}$} & No & 2296 & 93,0 \\
\hline & $\mathrm{Si}$ & 174 & 7,0 \\
\hline \multirow{2}{*}{$\begin{array}{l}\text { Episiotomía selectiva en } \\
\text { primíparas }\end{array}$} & No & 394 & 43,5 \\
\hline & $\mathrm{Si}$ & 512 & 56,5 \\
\hline \multirow{3}{*}{ Posición en el parto } & Sentada & 174 & 7,0 \\
\hline & Cuclillas & 23 & 0,9 \\
\hline & Acostada (supino) & 2284 & 92,1 \\
\hline \multirow{3}{*}{$\begin{array}{l}\text { Manejo activo del trabajo de } \\
\text { parto después de la } 41 \\
\text { semana de gestación }\end{array}$} & Espontáneo & 29 & 80,5 \\
\hline & Inducido & 5 & 13,9 \\
\hline & Cesárea electiva & 2 & 5,6 \\
\hline \multirow{3}{*}{$\begin{array}{l}\text { Manejo activo del } \\
\text { alumbramiento }\end{array}$} & $\mathrm{Si}$ & 1812 & 73,5 \\
\hline & No & 652 & 26,4 \\
\hline & Otros ocitócicos & 3 & 0,1 \\
\hline
\end{tabular}


Tabla 3. Prácticas en el cuidado de complicaciones durante la gestación y el parto

\begin{tabular}{cccc}
\hline \multicolumn{1}{c}{ Prácticas } & & Frecuencia & $\%$ \\
\hline $\begin{array}{c}\text { Corticoides antenatales para } \\
\text { promover la maduración fetal en } \\
\text { alto riesgo de parto pretérmino } \\
\text { embarazadas con alto riesgo de } \\
\text { parto pretérmino }\end{array}$ & $\begin{array}{c}\text { Incompleto } \\
\text { Completo }\end{array}$ & 9 & 7,7 \\
\hline $\begin{array}{c}\text { Administración de antibióticos } \\
\text { profilácticos en la cesárea }\end{array}$ & Ninguno & 19 & 17,1 \\
\hline $\begin{array}{c}\text { Uso de sulfato de magnesio en } \\
\text { preeclampsia severa }\end{array}$ & No & 323 & 16,2 \\
\hline Uso de sulfato de magnesio en & No & 205 & 38,2 \\
eclampsia & Si & 13 & 76,5 \\
\hline
\end{tabular}

Tabla 4. Prácticas en el cuidado del recien nacido

\begin{tabular}{cccc}
\hline & Prácticas & Frecuencia & $\%$ \\
\hline \multirow{2}{*}{ Lactancia materna } & Lactancia materna exclusiva & 2115 & 99,1 \\
& Lactancia materna y artificial & 16 & 0,8 \\
& Lactancia artificial & 3 & 0,1 \\
\hline \multirow{2}{*}{$\begin{array}{c}\text { Alojamiento conjunto } \\
\text { madre-hijo }\end{array}$} & Hospitalizado & 232 & 8,0 \\
& Alojamiento conjunto & 2643 & 91,2 \\
& Otro hospital & 23 & 0,8 \\
\hline
\end{tabular}

DISCUSIÓN

\section{Gestación}

En la población del estudio un porcentaje bajo pero representativo (20,6 \%) no presentó su carné materno-perinatal, al ingreso para la atención del parto, lo que limitó la información que sobre la evolución de la gestación pudo disponer el equipo de salud que realizó la atención, ya el carné perinatal, es una herramienta útil para la referencia y contrareferencia entre los servicios de salud, así como una estrategia que genera empoderamiento en la familia gestante. Esta práctica es recomendada por algunos estudios (4) por el sentimiento que genera en las mujeres de estar controladas por el personal de salud.

La indicación de la suplementación con hierro y ácido fólico es una práctica 
utilizada en un alto porcentaje de las gestantes, (87,3\%). Sin embargo, teniendo en cuenta que su uso rutinario mejora los índices hematológicos y disminuye de manera significativa los defectos del tubo neural cuando se utiliza oportuna y adecuadamente en las gestantes (5), se recomienda fortalecer el suministro y adherencia a su ingesta en la totalidad del grupo de mujeres gestantes.

Un alto porcentaje de mujeres atendidas en los servicios no fueron beneficiadas de esta práctica, ni antes (30,5\%) ni después (34\%) de la semana 20 de gestación, a pesar de que la tamización para sífilis se debe realizar a todas las gestantes y como mínimo, como un comportamiento óptimo institucional a por lo menos el $95 \%$ de las afiliadas al régimen subsidiado, meta que también ha establecido la Secretaría Distrital de Salud para la población participante vinculada; sin embargo. Hay que motivar a las instituciones a cumplir con la normatividad y garantizar el derecho que tiene el binomio madre-hijo a esta práctica que evitará morbilidad severa a largo plazo en la mujer y secuelas fatales, graves o discapacitantes en el feto y recién nacido (6).

A pesar de que un alto porcentaje de las gestantes asistió a por lo menos cinco controles prenatales la tamización de bacteriuria se realizó a cerca del 70 $\%$, por lo que se hace necesario profundizar en las causas por las cuales este examen no se realiza de manera rutinaria a todas las embarazadas, toda vez que se ha demostrado que el tratamiento de las bacteriurias asintomáticas disminuye el riesgo de pielonefritis y posiblemente reduce el número de partos prematuros (7).

Trabajo de parto

En las instituciones participantes no se favorece el acompañamiento durante el trabajo de parto en el $58 \%$ de los casos, a pesar de que ésta práctica ha demostrado disminución en la utilización de analgesia para el parto, en el número de partos instrumentados, en la reducción de la duración del trabajo de parto, aumento en la práctica de lactancia materna, de manera especial si la realiza una persona externa a la institución y el apoyo se inicia tempranamente (8). Teniendo en cuenta que se requiere una voluntad administrativa y del personal de salud, más que cambios infraestructurales, es importante que se orienten estrategias institucionales que garanticen esta práctica.

La utilización en las instituciones participantes del enema evacuante durante el trabajo de parto y el rasurado perineal de rutina para la preparación del parto fue muy baja (0,6 \% para el enema y 7,0 \% para el rasurado), comportamiento que se debe mantener y reforzar ya que son prácticas que han demostrado ser 
inefectivas además de molestas y no reducen el riesgo de infección puerperal (9) ni de la episiorrafia (10).

La episiotomía selectiva en primíparas, es una práctica indiscriminada que aún se utiliza con bastante frecuencia en las instituciones participantes (56,5 $\%)$, por lo que se debe difundir entre los profesionales los beneficios de realizar este procedimiento de manera selectiva, ya que este procedimiento no disminuye la incidencia de desgarro perineal y si aumenta la frecuencia de desgarros graves (11).

En relación con la posición en el parto se encontró que la más frecuente es la posición supina (92,1\%) por lo que se recomienda fomentar el parto institucional en posiciones más fisiológicas y cómodas para las gestantes (vertical, cuclillas o sentadas), ya que el parto en posición supina es más doloroso y ocasiona alteraciones en la fetocardia, además de que se requiere mayor utilización de fórceps (12).

El manejo activo del trabajo de parto a partir de la 41 semana de gestación es una práctica recomendada por los expertos (13), sin embargo su utilización a nivel institucional es muy bajo (de las maternas que lo requerían, el 80,5 \% no lo recibió), indicando la necesidad de difundir entre los profesionales de la salud los beneficios que genera su uso en la atención de las gestantes y particularmente en la calidad de vida de los recién nacidos.

El manejo activo del alumbramiento incluye el suministro de ocitócicos una vez ha salido el hombro o la cabeza del recién nacido, acompañado de masaje en el fondo uterino y tracción controlada del cordón umbilical hasta el alumbramiento. Fue medido por los investigadores solamente como la aplicación del ocitócico, hallándose un uso insuficiente (73,5 \%) de esta práctica, teniendo en cuenta que la literatura científica demostró su beneficio en términos de reducción de pérdida de sangre, hemorragia posparto y otras complicaciones graves del tercer estadio del trabajo de parto (hemorragia puerperal y hemorragia puerperal severa), aunque se presentan efectos secundarios desagradables como náusea y vómito, e hipertensión cuando se usa ergometrina (14). Es necesario resaltar en este punto que la hemorragia posparto se constituyó en la segunda causa de mortalidad materna en el año 2004 en la ciudad de Bogotá según información obtenida del Sistema de Vigilancia Epidemiológica de la Secretaría Distrital de Salud de Bogotá en el año 2005.

Tratamiento de complicaciones de la gestación y el parto 
La administración de corticoides para inducir maduración fetal en el alto riesgo de parto pretérmino, suministrado por lo menos 24 horas antes del nacimiento, reduce en el prematuro la mortalidad, el síndrome de dificultad respiratoria y la hemorragia intraventricular y el riesgo de enterocolitis necrotizante, además de mejorar la respuesta al surfactante utilizado en el periodo posnatal (15). Sin embargo, de las gestantes que tuvieron parto pretérmino y que cumplían con las condiciones para recibir el tratamiento, solamente el 51,6 \% accedieron a éste. Su implementación es fundamental si se tiene en cuenta que el síndrome de dificultad respiratoria fue en el 2004 la segunda causa básica de mortalidad perinatal en la ciudad de Bogotá, según la información de estadísticas vitales de la Secretaría Distrital de Salud en el año 2005.

Los antibióticos profilácticos se administraron en el 61,2 \% de las gestantes sometidas a cesárea, por lo que se deben generar políticas institucionales para la utilización de esta práctica ya que reduce en dos terceras partes la endometritis en cesárea programada y en tres cuartas partes en cesárea no programada (16).

Del total de gestantes a quienes se les diagnosticó preeclampsia severa o eclampsia al ingreso a la institución solamente 27,1 \% y 20,0 \%, respectivamente, recibieron tratamiento con sulfato de magnesio, lo que representa muy baja adherencia a la guía de atención de las complicaciones hipertensivas asociadas al embarazo del Ministerio de Protección Social (17), y que no responde a la evidencia mundial existente, que demuestra los beneficios de este medicamento en el manejo de la preeclampsia (18) y la eclampsia (19).

Para el 2004, según la información de estadísticas vitales de la Secretaría Distrital de Salud, los trastornos hipertensivos asociados a la gestación se constituyeron en la primera causa de mortalidad materna en la ciudad de Bogotá. Por las razones expuestas se hace necesario difundir y asegurar el cumplimiento de esta práctica en las instituciones de salud de la ciudad.

Cuidados del recién nacido

La lactancia materna exclusiva y sin restricciones, se fomentó en un 99,1 \% de las mujeres. La utilización de esta práctica ha demostrado una reducción significativa de la mortalidad infantil y las infecciones gastrointestinales (20).

En lo relacionado con el alojamiento conjunto madre-hijo, entendido como el contacto piel a piel durante la primera media hora del parto vaginal (o al menos durante la primera hora después del parto), y por lo menos durante 30 minutos (21), se favoreció en un 91,2 \% de los casos y en un 8 \% no se hizo 
debido a hospitalización del recién nacido. El contacto piel a piel prolonga la duración de la lactancia materna, el apego y la supervivencia del bebé, por lo que se debe continuar esta práctica hospitalaria.

Las evidencias clínicas existentes, la normatividad nacional para la atención de maternas y recién nacidos, así como las políticas dirigidas a la reducción de la mortalidad materna y perinatal en el mundo y en el país (23), sugieren el desarrollo de metodologías efectivas que garanticen la adherencia a normas y guías de atención, así como de decisiones administrativas y gerenciales que faciliten su cumplimiento.

Es importante divulgar dentro del personal que brinda atención a gestantes y neonatos, aquellas prácticas que basadas en la evidencia se consideran beneficiosas, así como aquellas que se han identificado como perjudiciales o inefectivas, para fomentar el uso de las primeras y eliminar la práctica de las últimas. Se requiere sensibilizar al personal de salud sobre la importancia de esta información para el mejoramiento de la calidad de la atención y la toma de decisiones en salud pública.

Agradecimientos. A Fred Lozano, Javier Bonilla, María Fernanda Beltrán y Raúl Mendoza, así como la colaboración del personal de salud y el apoyo de las gerencias de los hospitales Simón Bolívar, San Blas y Rafael Uribe Uribe, quienes hicieron posible la realización del presente estudio.

\section{REFERENCIAS}

1. Enkin M, Keirse M, Neilson J, Crowther C, Duley L, Hodnet H, Hofmeyr J. A Guide to Effective Care in Pregnancy and Childbirth. 3d ed, Oxford; Oxford University Press; 2000.

2. World Health Organization, Department of Reproductive Health and Research, UNDP/ UNFPA/WHO/World Bank Special Programme of Research, Development and Research Training in Human Reproduction. The WHO Reproductive Health Library No 5. Geneva: World Health Organization; 2002.

3. Sistema Informático Perinatal, Manual Resumido. Centro Latinoamericano de Perinatología y Desarrollo Humano. Publicación Científica del CLAP 1524. Montevideo, Uruguay; Julio 2003.

4. Biblioteca virtual de salud [Internet]. Disponible en: http://perinatal.bvsalud.org/html/ es/home.html Consultado Agosto de 2005.

5. Mahomed K Suplementación con hierro y folatos en el embarazo (Revisión Cochrane 
234 REVISTA DE SALUD PÚBLICA · Volumen 8 (3), Noviembre 2006

traducida). En: The Cochrane Library, Issue 1, Oxford. Update Software; 2002. 6. Walter GJA. Antibióticos para la sífilis diagnosticada durante el embarazo (Revisión Cochrane traducida) En: The Cochrane Library, Issue 4. Chichester, UK: John Wiley y Sons, Ltd.; 2003.

7. Smaill F. Tratamiento antibiótico para la bacteriuria asintomática en el embarazo (Revisión Cochrane traducida). In: The Cochrane Library, Issue 1, Oxford. Update Software; 2002.

8. Hodnett ED, Gates S, Hofmeyr GJ, Sakala C. Apoyo continuo para la mujer durante el parto (Revisión Cochrane tarducida). En: The Cochrane Library, Issue 4, Chichester, UK: John Wiley y Sons, Ltd.; 2003.

9. Cuervo LG, Rodriguez MN, Delgado MB. Enemas during labor (Cochrane Review) In: The Cochrane Library, 1. Oxford: Update Software; 2001.

10. Basevi V, Lavender T. Routine perineal shaving on admission in labour (Cochrane Review) In: The Cochrane Library, 1. Oxford: Update Software; 2001

11. Liljstrand J. Políticas de episiotomía en partos vaginales: Comentarios de la BSR (última revisión: 8 de julio de 2002). La Biblioteca de Salud reproductiva de la OMS, No. 7. Oxford: Update Software Ltd.; 2004.

12. Gupta JK, Hofmeyr GJ. Posición de la mujer durante el periodo expulsivo del trabajo de parto (Cochrane Review). In: The Cochrane Library, Issue 2. Oxford: Update Software; 2005.

13. Crowley P. Intervenciones para prevenir o mejorar el resultado del parto a término o postérmino (Revisión Cochrane traducida) En: The Cochrane Library, Issue 4. Chichester, UK: John Wiley y Sons, Ltd.; 2003.

14. Prendiville WJ, Elbourne D, McDonald S. Conducta activa versus conducta expectante en el alumbramiento (Revisión Cochrane traducida). The Cochrane Library, Issue 4. Chichester, UK: John Wiley y Sons, Ltd.; 2003.

15. Crowley P. Corticoesteroides con fines profilácticos para el parto prematuro (Revisión Cochrane traducida). The Cochrane Library, Issue 4. Chichester, UK: John Wiley y Sons, Ltd; 2003.

16. Smaill F, Hofmeyr GJ. Profilaxis antibiótica para la operación cesárea (Revisión Cochrane traducida). In: The Cochrane Library, Issue 1. Oxford: Update Software; 2002.

17. Normas técnicas y guías de atención en salud. Resolución 412 de 2000. Ministerio de Salud, Colombia; 2000.

18. Duley L, Gülmezoglu AM, Henderson-Smart D. Sulfato de magnesio y otros anticonvulsivos en mujeres con preeclampsia (Revisión Cochrane traducida). En: The Cochrane Library, Issue 4. Chichester, UK: John Wiley y Sons, Ltd.; 2003

19. Atallah AN. Tratamiento anticonvulsivo para la eclampsia: Comentario de la BSR. Chichester, UK: John Wiley y Sons, Ltd.; 2003.

20. De Onis M. Duración óptima de la lactancia materna exclusiva: Comentario de la BSR (última revisión: 11 de noviembre de 2002). La biblioteca de salud reproductiva de la OMS, No 7. Oxford: Update Software Ltd.; 2004.

21. Organización Mundial de la Salud. División de salud y desarrollo del niño. Pruebas científicas de los diez pasos hacia una lactancia natural. Ginebra; 1998. 